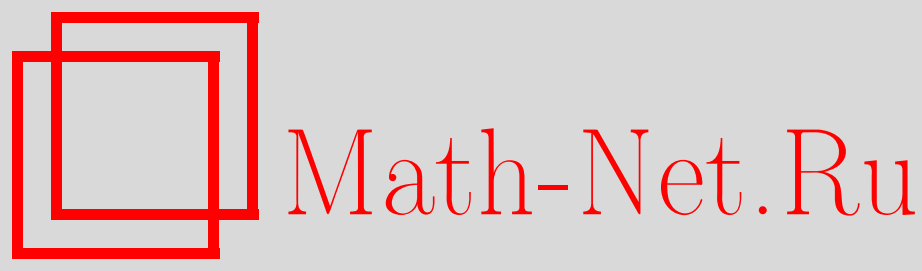

И. А. Дьяконов, М. В. Комарова, М. Ю. Налимов, Исследование температурных функций Грина графеноподобных систем в полупространстве, ТМФ, 2017, том 190, номер 3, 426-439

DOI: https://doi.org/10.4213/tmf9119

Использование Общероссийского математического портала Math-Net.Ru подразумевает, что вы прочитали и согласны с пользовательским соглашением http://www . mathnet.ru/rus/agreement

Параметры загрузки:

IP : 54.162 .27 .143

26 апреля 2023 г., 15:57:29

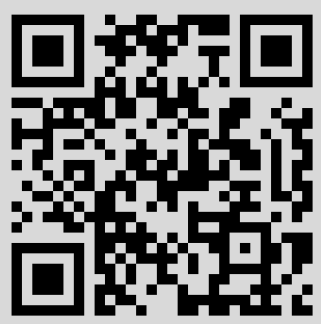




\title{
ИССЛЕДОВАНИЕ ТЕМПЕРАТУРНЫХ ФУНКЦИЙ ГРИНА ГРАФЕНОПОДОБНЫХ СИСТЕМ В ПОЛУПРОСТРАНСТВЕ
}

\begin{abstract}
Для исследования электронных свойств полубесконечной двумерной решетки графена при заданной температуре рассмотрен формализм температурных функций Грина. При наиболее общих предположениях об устройстве границы графена вычислен пропагатор соответствующей диаграммной техники. Полученный пропагатор выдерживает предельные переходы между физически различными состояниями границы системы - аценовым краем и граничным условием в приближении “бесконечной массы", а также корректно описывает задачу, в которой электрон-дырочная симметрия нарушена вследствие наличия внешнего потенциала, приложенного к границе графена. Использование пропагато$\mathrm{pa}$, его аналитические свойства и особенности вычислений с ним проиллюстрированы на примере расчета зависимости электронной плотности от расстояния до границы системы.
\end{abstract}

Ключевые слова: температурные функции Грина, графен, квантово-полевая теория возмущений, граничные условия.

DOI: https://doi.org/10.4213/tmf9119

\section{1. ВВЕДЕНИЕ}

Описание электронных свойств графена, начавшееся в 40-е годы XX века [1], получило мощный толчок к развитию в связи с успешными экспериментами по исследованию его зонной структуры [2] в 70-90-х годах и снова - после открытия простого способа получения монослоя графита [3].

Теоретическое описание электронных свойств системы удобно проводить в коллективных переменных, описывающих распространение квазичастиц в каждой из двух подрешеток Браве кристаллической структуры графена. В главном порядке

Работа выполнена при поддержке Санкт-Петербургского государственного университета (исследовательский грант 11.38.185.2014).

${ }^{*}$ Санкт-Петербургский государственный университет, Санкт-Петербург, Россия. E-mail: m.komarova@spbu.ru, frommarina@mail.ru,m.nalimov@spbu.ru, myunalimov@gmail.com, tanatos@gmail.com 


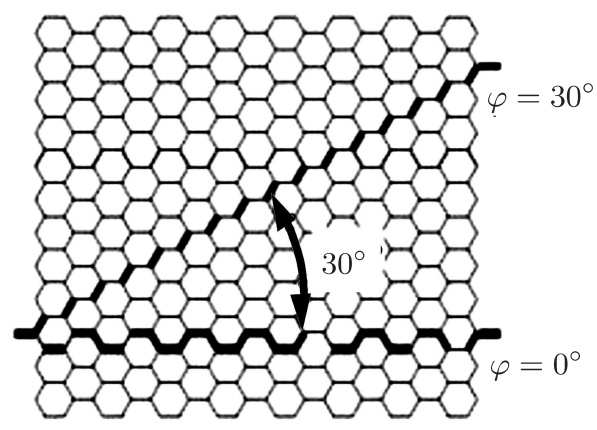

Рис. 1. Типы граничных условий для графена в полупространстве.

теории возмущений гамильтониан системы электронов в точности описывает динамику двумерных свободных релятивистских безмассовых фермионов. Это обуславливает важность применения в данной задаче квантово-полевого формализма, поскольку он поставляет готовый математический аппарат для исследования статистических свойств таких частиц.

Для построения последовательной теории возмущений в такой науке, однако, недостаточно переформулировать известные квантово-полевые результаты, касающиеся ультрарелятивистских ферми-систем. Тот факт, что движение электронов в графене ограничено плосткостью графена, приводит к очевидным и значительным отличиям в технике расчетов [4]. Влияние одномерной границы двумерного графена в полупространстве также не сводится к известным квантово-полевым результатам, и соответствующие эффекты являются объектом современных исследований [5].

Целью настоящей работы является построение пропагатора в полупространстве, подходящего для реализации диаграммных методов Фейнмана и использования ренормализационной группы. Вычисленный в рамках стандартного квантово-полевого подхода [5] пропагатор демонстрирует при некоторых типах граничных условий сингулярное поведение, затрудняющее его применение в регулярной теории возмущений. Поэтому для описания электронных свойств графена мы используем формализм температурных функций Грина. При этом мы контролируем аналитические свойства пропагатора в обсуждаемом спектре физических граничных условий в полупространстве. Использование построенного пропагатора мы иллюстрируем примером вычисления граничных эффектов для электронной плотности системы.

В рассмотренной нами модели граница полубесконечного графена ориентирована под произвольным углом $0^{\circ}<\varphi \leqslant 30^{\circ}$ к выбранной оси симметрии решетки (см. рис. 1). Граничные условия в данной задаче строятся, как известно, на основе двух принципов: условия отсутствия протекания заряда через границу и принципа электрон-дырочной симметрии [6]. При этом случай $\varphi=0$ (так называемый фенантреновый край - граница в форме кресла, "armchair") связывают с образованием вблизи границы запрещенных зон.

Вопрос выбора правильных граничных условий в графене продолжает широко обсуждаться. Часто используются граничные условия типа Берри-Мандрагона, также известные как "MIT bag" или граничные условия в приближении "бесконечной массы" [5]. В работе [7] было показано, что переход от граничных условий 


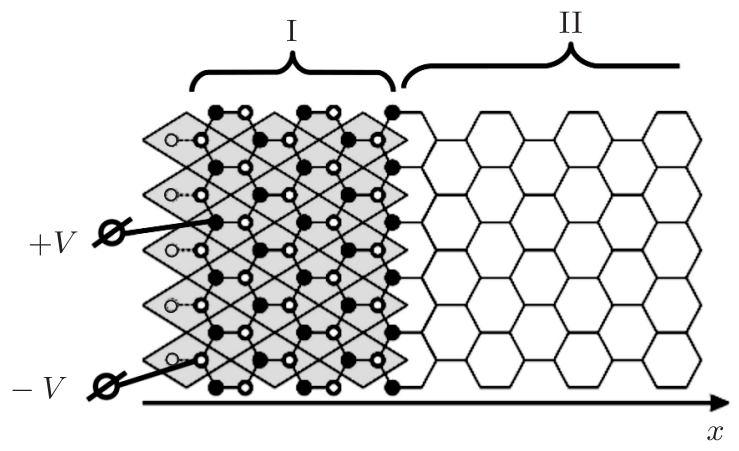

Рис. 2. Полуограниченная решетка графена (II) с границей (I). В граничном слое к атомам, помеченным черным, приложен положительный потенциал, а к атомам, помеченным белым, - отрицательный [7].

в приближении "бесконечной массы" к условию аценового края (разрез в форме зигзага, $\varphi=30^{\circ}$ ) можно выполнить непрерывно, вводя вспомогательный параметр $\vartheta \in[0, \pi / 2]$, нарушающий электрон-дырочную симметрию при $\vartheta \neq 0$ и $\vartheta \neq \pi / 2$. Такой модели при $0<\vartheta<\pi / 2$ можно сопоставить физическую систему, в которой на границе полупространства к графену с разрезом при $\varphi=30^{\circ}$ приложен периодический в пространстве электрический потенциал (см. рис. 2 и работу [7]). Наличие потенциала нарушает электрон-дырочную симметрию; с его увеличением плавно меняется значение $\vartheta$; изменение потенциала в интервале от нуля до бесконечности плавно преобразует зигзагоподобное граничное условие в условие типа приближения "бесконечной массы".

Рассмотрение аценового края $\vartheta=\pi / 2$ обычно вызывает аналитические сложности в квантово-полевом формализме, поэтому важный результат настоящей работы состоит в том, что построенный нами пропагатор может корректно использоваться при произвольных значениях $\vartheta$.

Прямым способом получения физически значимых результатов с помощью пропагатора является вычисление электронной плотности. Некоторые технические сложности, выражающиеся в наличии ультрафиолетовых (УФ) расходимостей, связаны с корректным учетом сдвига дираковского "подвала" в термодинамической постановке задачи по сравнению с квантово-полевой формулировкой. Мы приводим подробный анализ появления расходимостей такого типа и обсуждаем корректное обращение с ними.

Работа организована следующим образом. В разделе 2 вводится гамильтониан рассматриваемой задачи, граничные условия, обсуждаются особенности формализма температурных функций Грина. В разделе 3 обсуждается специфика учета граничных условий. В разделе 4 в смешанном $\left(x, p_{y}, \omega_{n}\right)$-представлении вычисляется пропагатор этой модели - двухчастичная свободная температурная функция Грина; обсуждается выражение для профиля электронной плотности. В разделе 5 обсуждается суммирование по мацубаровским частотам $\omega_{n}$ и его реализация в случае построенной температурной функции Грина. В разделе 6 на примере модельного интеграла обсуждается структура выражений, получающихся при мацубаровском 
суммировании, аналитические свойства пропагатора и физическая причина появления УФ-расходимостей в данной модели. В разделе 7 приводится результат для профиля электронной плотности системы. В заключении мы суммируем полученные результаты.

\section{2. ПОСТАНОВКА ЗАДАЧИ В ТЕРМИНАХ ТЕМПЕРАТУРНЫХ ФУНКЦИЙ ГРИНА}

Электронные свойства графена описываются динамикой квазичастиц на двух подрешетках Браве его кристаллической структуры. Одночастичный гамильтониан электронов проводимости разлагается в ряд в окрестности двух точек $K_{+}$и $K_{-}$, лежащих в зоне Брюллиэна. В этих точках реализуется минимум гамильтониана (далее мы будем использовать традиционный термин “долина" для окрестности каждой такой точки). Разложение, соответствующее свободной от взаимодействия модели, имеет в точности вид гамильтониана двумерных релятивистских фермионов [1], [3]:

$$
\widehat{H}_{ \pm}=\hbar v_{\mathrm{F}}\left(-i \sigma_{2} \partial_{x} \pm i \sigma_{1} \partial_{y}\right) \text {. }
$$

Здесь $v_{\mathrm{F}}$ - скорость Ферми, $\sigma_{1}, \sigma_{2}$ - матрицы Паули в пространстве подрешеток (роль спинорного индекса релятивистских фермионов играет индекс, определяющий подрешетку). Знаки \pm соответствуют разложению гамильтониана в точках $K_{ \pm}$.

Для описания многочастичной системы вводится квантовое поле $\hat{\psi}(x, y)$ в двумерном $(x, y)$-пространстве. Комплексное поле $\hat{\psi}$ имеет две компоненты, соответствующие двум подрешеткам. Каждая компонента, в свою очередь, имеет спинорные индексы, которые отражают фермионные свойства соответствующих квазичастиц. Поля удовлетворяют стандартным антикоммутационным соотношениям $\left[\hat{\psi}, \hat{\psi}^{\dagger}\right]_{+}=1$, где $\hat{\psi}^{\dagger} \equiv \hat{\psi}^{* \mathrm{~T}}$.

Чтобы описать при заданной температуре равновесный ансамбль системы таких частиц, вычисляется статистическая сумма

$$
\Theta=\operatorname{Tr} e^{\beta \hat{\psi}^{\dagger}(\mu-\widehat{H}) \hat{\psi}},
$$

где $\beta$ - обратная больцмановская температура, $\mu$ - химический потенциал; процедура взятия следа, кроме суммирования по всем индексам и интегрирования по координатам, включает в себя суммирование по долинам вокруг $K_{ \pm}$. В рамках формализма температурных функций Грина [8] это выражение преобразуется при помощи формулы Фейнмана-Каца к функциональному интегралу. Для этого в квантовые поля вводится дополнительная температурная переменная $\tau$, соответствующая квантово-механическому "мнимому времени"; предполагается, что эта переменная изменяется в интервале $(0, \beta)$.

Статистическая сумма принимает вид

$$
\begin{gathered}
\Theta=\sum_{ \pm} \int \mathcal{D} \psi \mathcal{D} \psi^{\dagger} e^{-S_{\beta}} \\
S_{\beta}=\int_{0}^{\beta} d \tau \int_{0}^{\infty} d x \int_{-\infty}^{\infty} d y \psi^{\dagger}(x, y, \tau)\left(\sigma_{0}\left(\partial_{\tau}-\mu\right)+\widehat{H}_{ \pm}\right) \psi(x, y, \tau),
\end{gathered}
$$


где $\sigma_{0}$ - единичная матрица. На поля при этом налагаются антипериодические условия по переменной $\tau$, соответствующие решениям фермионного типа:

$$
\left.\psi\right|_{\tau=0}=-\left.\psi\right|_{\tau=\beta}
$$

В терминах мацубаровских частот это означает, что $\psi$ представляется в виде ряда по частотам $\omega_{n}$ (где $n$ - целое),

$$
\psi(x, y, \tau)=\sum_{\omega_{n}} e^{-i \omega_{n} \tau} \psi\left(x, y, \omega_{n}\right), \quad \omega_{n}=\frac{\pi}{\beta}(2 n+1) .
$$

Температурная функция Грина (пропагатор) $G\left(x, x^{\prime}, y, y^{\prime}, \tau, \tau^{\prime}\right)$, соответствующая данному описанию, удовлетворяет, таким образом, уравнению

$$
\left(\sigma_{0}\left(\partial_{\tau}-\mu\right)+\widehat{H}_{ \pm}\right) G=\delta\left(x-x^{\prime}\right) \delta\left(y-y^{\prime}\right) \delta\left(\tau-\tau^{\prime}\right)
$$

с антипериодическими условиями по переменным $\tau, \tau^{\prime}$.

Удобно обезразмерить переменные, введя дополнительно к скорости Ферми $v_{\mathrm{F}}$ энергию Ферми $\epsilon_{\mathrm{F}}$. Отношение $x_{\mathrm{F}}=\hbar v_{\mathrm{F}} / \epsilon_{\mathrm{F}}$ имеет размерность длины, так что, используя параметр $x_{\mathrm{F}}$, можно обезразмерить переменные $x, x^{\prime}, y$ и $y^{\prime}$. Переменные $\tau$ и $\tau^{\prime}$ с размерностью обратной энергии естественно обезразмеривать с помощью величины $\epsilon_{\mathrm{F}}$. Если иное не оговорено особо, дальнейшие вычисления проводятся в безразмерных переменных.

\section{3. ГРАНИЧНЫЕ УСЛОВИЯ В ПОЛУПРОСТРАНСТВЕ}

Граничные условия для полей на границе полупространства $x=0$ могут быть записаны в матричном виде [6]

$$
\left.\left(\sigma_{0}+\sigma_{1} e^{-i \sigma_{2} \vartheta}\right) \psi(x, y, \tau)\right|_{x=0}=0 .
$$

Режим граничных условий в приближении "бесконечной массы" получается, если положить здесь $\vartheta=0$; при $\vartheta=\pi / 2$ получаются граничные условия типа зигзаг, причем в соответствии с работой [7] последнее, зигзагообразное, условие приемлемо с микроскопической точки зрения описывает граничные состояния при всех углах разреза, кроме $\varphi \rightarrow 0^{\circ}$. При произвольных значениях $\vartheta$ граничные условия (4) описывают модельную задачу, когда к граничным атомам одной подрешетки графена приложен электрический потенциал $V$, а к атомам второй подрешетки - такой же потенциал с противоположным знаком. Предполагается, что потенциал действует на $2 N$ рядов атомов вблизи границы (см. рис. 2). За подробностями сведе́ния задачи с граничным электрическим потенциалом $V$ к модели с граничными условиями типа зигзаг или к модели с условием "бесконечной массы" мы отсылаем читателя к работе [7]; хочется лишь отметить, что использованный в указанной работе формализм требует специфического порядка предельных переходов по $N$ и $V$ при рассмотрении обсуждаемых предельных случаев, а угол $\theta$ в этой работе отличается от используемого в работе [6] и параметра $\vartheta$ в (4) на $\pi / 2$.

Предполагается, что поля $\Psi$ затухают на больших расстояниях от границы (при $x,|y| \rightarrow+\infty)$ - соответствующее поведение обеспечивается переходом от плоских волн в квантовой механике к мнимому времени формализма температурных функций Грина. Заметим, что граничное условие и условие затухания в пространстве фиксируют функциональное пространство, описывающее рассматриваемую модель. 


\section{4. ВЫЧИСЛЕНИЕ ПРОПАГАТОРА И ЕГО СВЯЗЬ С ПЛОТНОСТЬЮ КВАЗИЧАСТИЦ ВБЛИЗИ ГРАНИЦЫ}

Функциональное пространство, которому принадлежит пропагатор, определяется функциональным пространством полей $\psi[9]$. Поэтому кроме условия (2) антипериодичности по $\tau, \tau^{\prime}$ уравнение (3) для пропагатора должно быть дополнено граничным условием типа (4),

$$
\left.\left(\sigma_{0}+\sigma_{1} e^{-i \sigma_{2} \vartheta}\right) G\left(x, x^{\prime}, y-y^{\prime}, \tau-\tau^{\prime}\right)\right|_{x=0}=0,
$$

а также условием затухания пропагатора при удалении от границы. Несложно убедиться, что (5) эквивалентно следующему матричному представлению функции Грина:

$$
\left.G\right|_{x=0}=\left(\begin{array}{ll}
1 & 0 \\
0 & \varsigma
\end{array}\right)\left(\begin{array}{ll}
1 & 1 \\
1 & 1
\end{array}\right)\left(\begin{array}{cc}
A_{1} & 0 \\
0 & A_{2}
\end{array}\right), \quad \varsigma=-\frac{1+\sin \vartheta}{\cos \vartheta} .
$$

Решение удобно искать, совершив в (3) преобразование Фурье по трансляционноинвариантным переменным $y-y^{\prime}$ и $\tau-\tau^{\prime}$ :

$$
-\left(\sigma_{0}\left(i \omega_{n}+\mu\right)+i \sigma_{2} \partial_{x} \pm \sigma_{1} p_{y}\right) G=\delta\left(x-x^{\prime}\right)
$$

здесь и далее для фурье-образа функции не вводится специального обозначения, различие между различными представлениями пропагатора могут указываться явно в виде аргументов функции или подразумеваться по контексту.

Общее решение фундаментального уравнения (7) имеет вид

$$
G\left(x, x^{\prime}, p_{y}, \omega_{n}\right)=G_{1} \theta\left(x-x^{\prime}\right)+G_{2},
$$

где $G_{1,2}$ удовлетворяют однородному уравнению, соответствующему (7) с отброшенной правой частью. Их несложно найти:

$$
\begin{aligned}
& G_{i}=\widetilde{G}\left(\begin{array}{cc}
B_{i 1} & 0 \\
0 & B_{i 2}
\end{array}\right) e^{\varkappa x}+\sigma_{1} \widetilde{G} \sigma_{1}\left(\begin{array}{cc}
B_{i 3} & 0 \\
0 & B_{i 4}
\end{array}\right) e^{-\varkappa x}, \\
& \widetilde{G}=\left(\begin{array}{cc}
\frac{\mp p_{y}+\varkappa}{i \omega_{n}+\mu} & 1 \\
1 & \frac{\mp p_{y}-\varkappa}{i \omega_{n}+\mu}
\end{array}\right), \quad \varkappa=\sqrt{p_{y}^{2}-\left(i \omega_{n}+\mu\right)^{2}} .
\end{aligned}
$$

Параметры $B_{i j}\left(x^{\prime}\right)$ являются константами по переменной $x$; для их определения необходимо обратиться к граничному условию (5) и условию убывания функции $G$ на бесконечности. Это приводит к выражению

$$
\begin{aligned}
G\left(x, x^{\prime}, p_{y}, \omega_{n}\right)=\frac{1}{1-\gamma}( & \theta\left(x-x^{\prime}\right)\left(\widetilde{G} \Gamma e^{-\varkappa\left(x-x^{\prime}\right)}-\sigma_{1} \widetilde{G} \Gamma \sigma_{1} e^{\varkappa\left(x-x^{\prime}\right)}\right)+ \\
& \left.+\sigma_{1} \widetilde{G} \Gamma \sigma_{1} e^{\varkappa\left(x-x^{\prime}\right)}-\widetilde{G} \Delta_{M} \sigma_{1} \Gamma \sigma_{1} e^{-\varkappa\left(x+x^{\prime}\right)}\right) .
\end{aligned}
$$

Здесь для удобства введены следующие обозначения:

$$
\begin{gathered}
\Gamma=\left(\begin{array}{ll}
\gamma & 0 \\
0 & 1
\end{array}\right), \quad \gamma=\frac{ \pm p_{y}+\varkappa}{ \pm p_{y}-\varkappa}, \quad \Delta_{M}=\left(\begin{array}{cc}
\Delta_{1} & 0 \\
0 & \Delta_{2}
\end{array}\right), \\
\Delta_{1}=\frac{i \omega_{n}+\mu+\varsigma\left( \pm p_{y}+\varkappa\right)}{i \omega_{n}+\mu+\varsigma\left( \pm p_{y}-\varkappa\right)}, \quad \Delta_{2}=\frac{ \pm p_{y}-\varkappa+\varsigma\left(i \omega_{n}+\mu\right)}{ \pm p_{y}+\varkappa+\varsigma\left(i \omega_{n}+\mu\right)} .
\end{gathered}
$$


Последнее слагаемое в $(9)$, не являющееся трансляционно-инвариантным по $x-x^{\prime}$ и пропорциональное $\Delta_{M}$, содержит все вклады, связанные с граничными эффектами. При этом зигзагоподобное граничное условие (при $\varsigma \rightarrow \infty)$ характеризуется конечной матрицей $\Delta_{M}$, как и граничные условия при остальных значениях $\vartheta$. Обратим внимание, что это позволяет осуществлять непрерывный переход к пределу $\vartheta=\pi / 2$ в пропагаторе.

Мы построили пропагатор в смешанном $\left(x, p_{y}, \omega_{n}\right)$-представлении. Он затухает с ростом $x$ и существует при любых значениях $\vartheta \in[0, \pi / 2]$. Это позволяет использовать его для построения регулярной теории возмущений и разнообразных диаграммных вычислений, например для исследования фазовых переходов в модели со взаимодействием. Условие затухания граничных эффектов на больших расстояниях от границы, использованное при получении (9), определяется знаком $\varkappa>0$, что, в свою очередь, определяет лист двулистной комплексной поверхности, на которой задана функция $\varkappa\left(p_{y}\right)$ в $(8)$ и решение $(9)$. При вычислениях с таким пропагатором, однако, требуется учитывать специфику поставленной задачи. Это мы проиллюстрируем на примере вычисления зависимости электронной плотности в графене от расстояния до границы полупространства.

Используя функцию Грина (9), можно вычислить профиль линейной электронной плотности как функцию расстояния до границы системы $x=0$ :

$$
n(x)=-\left.\operatorname{Tr} G\right|_{\substack{x=x^{\prime}, y=y^{\prime} \\ \tau=\tau^{\prime}-0}}=-\sum_{ \pm, n} \int_{-\infty}^{\infty} \frac{d p_{y}}{2 \pi \beta} \operatorname{Tr} G(x, x) ;
$$

в данном выражении суммирование ведется по “долинам" $K_{ \pm}$и по мацубаровским частотам $\omega_{n}=\pi(2 n+1) / \beta$ с целыми $n$. Подставляя $(9)$, вычисляя след по подрешеточному индексу и производя суммирование по "долинам", несложно получить, что

$$
n(x)=-g_{s} \sum_{n} \int_{-\infty}^{\infty} \frac{d p_{y}}{\pi \beta}\left(\frac{i \omega_{n}+\mu}{\varkappa}-\frac{p_{y}^{2} e^{-2 \varkappa x}}{\varkappa\left(i \omega_{n}+\mu-\varkappa \operatorname{ctg} \vartheta\right)}\right)
$$

где $g_{s}=2-$ спиновое вырождение ферми-частиц. Первое слагаемое соответствует системе квазичастиц при отсутствии границ, второе описывает затухающий вклад граничных эффектов в плотность квазичастиц.

Прежде чем вычислять электронную плотность (11), следует обсудить особенности процедуры мацубаровского суммирования.

\section{5. ВЫЧИСЛЕНИЕ СУММ ПО МАЦУБАРОВСКИМ ЧАСТОТАМ}

Корректное суммирование по $n$ должно приводить к ответам, которые получаются непосредственно в представлении $G\left(x, x^{\prime}, y, y^{\prime}, \tau-\tau^{\prime}\right)$. Эти соображения позволяют доопределить процедуру обратного фурье-преобразования от переменной $\omega_{n}$ к переменной $\tau$.

В рамках формализма температурных функций Грина уравнение для функции Грина в $(\tau, p)$-представлении имеет вид

$$
\left(\partial_{\tau}+\epsilon(p)\right) G\left(p, \tau-\tau^{\prime}\right)=\delta\left(\tau-\tau^{\prime}\right), \quad p=\left(\begin{array}{c}
p_{x} \\
p_{y}
\end{array}\right), \quad \tau, \tau^{\prime} \in(0, \beta)
$$


здесь $\epsilon(p)$ - спектр некоторого одночастичного гамильтониана. Решение этого уравнения $G=\theta\left(\tau-\tau^{\prime}\right) g_{1}+g_{2}$, где $g_{1,2}-$ решения соответствующего однородного уравнения, после наложения антипериодических граничных условий немедленно приводится к виду

$$
G=e^{-\epsilon\left(\tau-\tau^{\prime}\right)}\left(\theta\left(\tau-\tau^{\prime}\right)-\frac{1}{e^{\beta \epsilon}+1}\right)
$$

С другой стороны, в $\left(p, \omega_{n}\right)$-представлении функция Грина есть

$$
G\left(p, \omega_{n}\right)=\frac{1}{-i \omega_{n}+\epsilon}
$$

Сравнивая выражения (12) и (13), получим доопределение обратного фурье-преобразования:

$$
\frac{1}{\beta} \sum_{n} \frac{e^{-i \omega_{n}\left(\tau-\tau^{\prime}\right)}}{-i \omega_{n}+\epsilon}=e^{-\epsilon\left(\tau-\tau^{\prime}\right)}\left(\theta\left(\tau-\tau^{\prime}\right)-\frac{1}{e^{\beta \epsilon}+1}\right) .
$$

Предполагается, что левая и правая часть в последнем выражении выражены в безразмерных переменных. Заметим, что после дифференцирования соотношения (13) по $\epsilon$ ряд в левой части становится равномерно сходящимся.

Технически суммирование по $n$ с эквивалентным по отношению к (14) доопределением можно осуществить в виде контурного интегрирования по дополнительной переменной $p_{0}$ (см., например, статью [10]):

$$
\frac{1}{\beta} \sum_{n} F\left(i \omega_{n}\right)=\frac{1}{2 \pi i} \oint_{\gamma} d p_{0} f\left(p_{0}\right) F\left(p_{0}\right) .
$$

Это равенство справедливо, если функция $\beta f\left(p_{0}\right)$ имеет единичные вычеты в точках $i \omega_{n}$ и аналитична в остальной части комплексной плоскости переменной $p_{0}$. Таким свойством обладает, с точностью до знака, плотность числа состояний фермичастиц, поэтому

$$
f\left(p_{0}\right)=-\frac{1}{e^{\beta p_{0}}+1}+M
$$

где $M$ - произвольный вклад, аналитичный по $p_{0}$. Различный выбор величины $M$ соответствует различным способам доопределения левой части выражения (14). Замкнутый в пространстве $\bar{C}$ (в пространстве комплексных чисел со включением бесконечности) контур интегрирования $\gamma$ в (15) выбирается, как показано на рис. 3, где крестиками отмечены полюсы функции $f\left(p_{0}\right)$; контур охватывает их, обходя против часовой стрелки.

Несложно показать, что выбор

$$
f\left(p_{0}\right)=\theta\left(\tau-\tau^{\prime}\right)-\frac{1}{e^{\beta p_{0}}+1}
$$




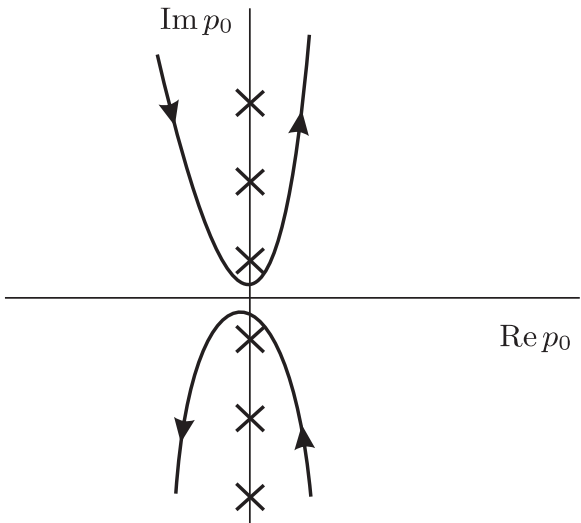

Рис. 3. Полюсы функции $f\left(p_{0}\right)$ и контур $\gamma$, охватывающий их.

эквивалентен доопределению (14). Действительно, рассмотрев в формуле (15) в качестве $F\left(p_{0}\right)$ выражение $\left(-p_{0}+\epsilon\right)^{-1} e^{-p_{0}\left(\tau-\tau^{\prime}\right)}$, деформируя контур $\gamma$ так, чтобы он охватывал полюс $p_{0}=\epsilon$, и вычисляя получившийся интеграл по вычетам, немедленно получаем (14).

\section{6. АНАЛИТИЧЕСКИЕ СВОЙСТВА ПРОПАГАТОРА, ЧАСТИЦЫ И АНТИЧАСТИЦЫ, “ДИРАКОВСКИЙ ПОДВАЛ"}

Рассмотрим модельный интеграл

$$
I=\frac{1}{\beta} \sum_{n} \int_{-\infty}^{\infty} d p_{y} \frac{e^{-x K\left(i \omega_{n}+\mu\right)} e^{-i \omega_{n}\left(\tau-\tau^{\prime}\right)}}{K\left(i \omega_{n}+\mu\right)}, \quad x \geqslant 0, \quad K(z)=\sqrt{p_{y}^{2}-z^{2}}
$$

и проведем в нем суммирование по $n$ посредством контурного интегрирования, описанного в предыдущем разделе. Данное выражение достаточно простое, чтобы эквивалентность такого расчета доопределению (14) можно было видеть явно. С другой стороны, структура формулы (16) аналогична граничному (последнему) вкладу в (11), что позволяет выявить специфику аналогичных вычислений для (11), связанную с аналитической структурой пропагатора (9).

Так как в (11) нам потребуется вычислять выражение при $\tau<\tau^{\prime}$ (точнее, при $\left.\tau=\tau^{\prime}-0\right)$, воспользуемся соответствующим доопределением в выражениях (14) и (15).

Метод I (контурное интегрирование). После перехода от суммирования к контурному интегрированию с помощью (15), замены переменных $z=p_{0}+\mu$ и использования четности по $p_{y}$ получим

$$
I=-\frac{1}{\pi i} \oint_{\gamma^{\prime}} d z \int_{0}^{\infty} d p_{y} \frac{e^{-x K(z)} e^{-(z-\mu)\left(\tau-\tau^{\prime}\right)}}{\left(e^{\beta(z-\mu)}+1\right) K(z)}
$$




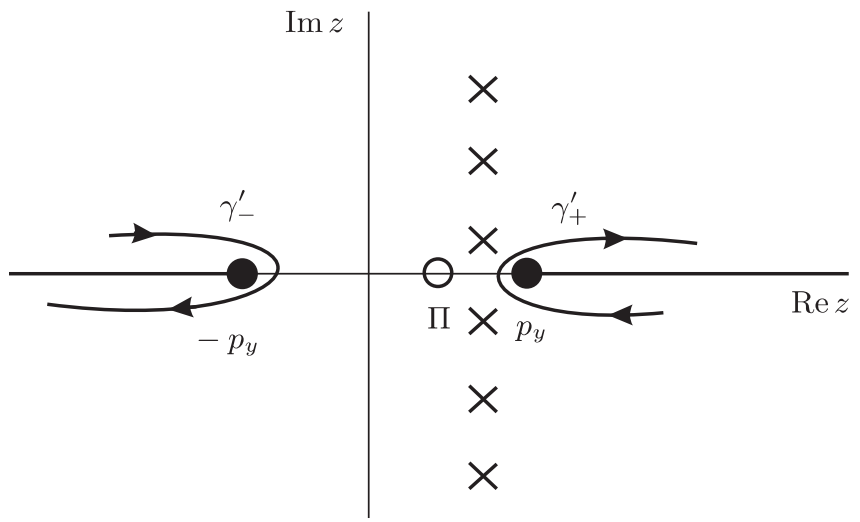

Рис. 4. Контур $\gamma^{\prime}$, полюсы и точки ветвления $\pm p_{y}$.

Контур $\gamma^{\prime}$ можно деформировать и приблизить его к краю разреза, как показано на рис. 4. Дальнейшее вычисление интеграла $I$ вдоль берегов разреза дает

$$
\begin{aligned}
\frac{1}{\pi i} \int_{0}^{\infty} d p_{y}\left(\int_{p_{y}}^{\infty}-\int_{-\infty}^{-p_{y}}\right) d z \frac{e^{-(z-\mu)\left(\tau-\tau^{\prime}\right)}}{e^{\beta(z-\mu)}+1} \frac{e^{-x K(z)}+e^{x K(z)}}{K(z)}= \\
=\frac{2}{\pi} \int_{0}^{\infty} d p_{y} \int_{p_{y}}^{\infty} d z\left(-\frac{e^{-(z-\mu)\left(\tau-\tau^{\prime}\right)}}{e^{\beta(z-\mu)}+1}+\frac{e^{(z+\mu)\left(\tau-\tau^{\prime}\right)}}{e^{-\beta(\mu+z)}+1}\right) \frac{\cos \left(x \sqrt{z^{2}-p_{y}^{2}}\right)}{\sqrt{z^{2}-p_{y}^{2}}} .
\end{aligned}
$$

Заменив порядок интегрирования на $\int_{0}^{\infty} d z \int_{0}^{z} d p_{y}(\cdot)$, а затем введя переменную $\alpha$ с помощью замены $p_{y}=z \sin \alpha$ и вычислив интеграл по $\alpha$, получим ответ в виде однократного интеграла, содержащего функцию Бесселя:

$$
I=-\int_{0}^{\infty} d z\left(\frac{e^{-(z-\mu)\left(\tau-\tau^{\prime}\right)}}{e^{\beta(z-\mu)}+1}-\frac{e^{(z+\mu)\left(\tau-\tau^{\prime}\right)}}{e^{-\beta(\mu+z)}+1}\right) J_{0}(x z) .
$$

Метод II (прямое суммирование мацубаровских частот). Чтобы проиллюстрировать эквивалентность нашего расчета доопределению (14), заметим, что выражение (16) можно просуммировать непосредственно с помощью соотношения (14). Для этого представим его в виде вспомогательного интеграла по переменной $p_{1}$ :

$$
I=\frac{1}{\beta} \sum_{n} \int_{-\infty}^{\infty} d p_{y} \int_{-\infty}^{\infty} \frac{d p_{1}}{2 \pi} \frac{2 e^{i p_{1} x} e^{-i \omega_{n}\left(\tau-\tau^{\prime}\right)}}{p_{1}^{2}+p_{y}^{2}-\left(i \omega_{n}+\mu\right)^{2}} .
$$

Переход к полярным координатам в двумерном пространстве $\left(d p_{1} d p_{y} \rightarrow z d z d \alpha\right)$ и вычисление интеграла по $\alpha$ приводит к выражению

$$
I=\int_{0}^{\infty} d z J_{0}(z x) \sum_{n} e^{-i \omega_{n}\left(\tau-\tau^{\prime}\right)}\left(\frac{1}{-i \omega_{n}+(z-\mu)}-\frac{1}{-i \omega_{n}-(\mu+z)}\right) .
$$

Пользуясь вспомогательной формулой (14) при $\tau<\tau^{\prime}$, получаем совпадающий с (18) ответ. 
Таким образом,

$$
\left.I\right|_{\tau=\tau^{\prime}-0}=-\int_{0}^{\infty} d z\left(\frac{1}{e^{\beta(z-\mu)}+1}-\frac{1}{e^{-\beta(\mu+z)}+1}\right) J_{0}(x z) .
$$

Так же, как в полученном нами ответе (19) для модельного интеграла, вычисление профиля плотности приводит к выражениям, в которых легко отождествить одни слагаемые со вкладом в статистическую сумму частиц, а другие - со вкладом античастиц с присущей им статистикой. Отличие связано не только с характерным видом знаменателей в (19), но и с различным поведением слагаемых в (18) по $\tau-\tau^{\prime}$, которое указывает, что экспоненциально убывающие по $\tau-\tau^{\prime}$ слагаемые относятся к частицам, а экспоненциально растущие - к античастицам.

Заметим, что, хотя интеграл (19) сходится при больших $z$, сходимость первого слагаемого обеспечивается экспоненциально убывающим при высоких энергиях распределением Ферми, в то время как второе слагаемое в скобках с ростом z стремится к единице. Такое поведение связано с формальным наличием в нашей системе сколь угодно высокоэнергетичных античастиц из "дираковского подвала".

Здесь следует сделать важное замечание. Физически в задачах, где исследуется плазма твердых тел, энергия дырок не может превышать значения химического потенциала. Первые слагаемые в (18) и в (19) содержат вклады частиц (при $z-\mu>0$ ) и вклады именно таких дырок $(0 \leqslant z \leqslant \mu)$. Вторые слагаемые в (18) и в (19) содержат вклад "нефизических" античастиц $(\mu+z>\mu)$. Происхождение такого вклада связано с тем, что исходная модель с гамильтонианом (1) получается путем разложения микроскопического гамильтониана в окрестности точек минимума в пространстве обратной решетки, и поэтому сама модель справедлива лишь в некоторой инфракрасной области. Так как математически структура гамильтониана твердотельной задачи соответствует газу релятивистских ферми-частиц, рассматривать его на всем интервале импульсов и применять известные результаты релятивистской квантовой теории весьма удобно. Это, однако, приводит к появлению в ответе вклада от "нефизических" античастиц.

Удобство метода I заключается в том, что нефизические вклады можно отделить с самого начала, поскольку вклад частиц и физических дырок соответствует интегралу по контуру $\gamma_{+}^{\prime}$, а нефизических античастиц - по контуру $\gamma_{-}^{\prime}$. Отбрасывание "нефизических" античастиц при вычислении профиля электронной плотности соответствует физическому обрезанию УФ-расходимостей в модели.

\section{7. ПРОФИЛЬ ЭЛЕКТРОННОЙ ПЛОТНОСТИ}

Осталось вычислить выражение (11), воспользовавшись развитыми в предыдущем разделе идеями. После перехода от суммирования по $n$ к интегрированию ответ естественным образом разбивается на слагаемые $n(x)=n_{0}+n_{\mathrm{b}}(x)$, где

$$
\begin{aligned}
n_{0} & =\frac{g_{s}}{2 \pi i} \oint_{\gamma} \frac{d p_{0}}{e^{\beta p_{0}}+1} \int_{-\infty}^{\infty} \frac{d p_{y}}{\pi} \frac{p_{0}+\mu}{\varkappa} \\
n_{\mathrm{b}}(x) & =-\frac{g_{s}}{2 \pi i} \oint_{\gamma} \frac{d p_{0}}{e^{\beta p_{0}}+1} \int_{-\infty}^{\infty} \frac{d p_{y}}{\pi} \frac{p_{y}^{2} e^{-2 \varkappa x}}{\varkappa\left(p_{0}+\mu-\varkappa \operatorname{ctg} \vartheta\right)} .
\end{aligned}
$$


Вклад $n_{0}$ соответствует электронной плотности в отсутствие граничных эффектов, всё влияние границы на свойства системы отражает вклад $n_{\mathrm{b}}(x)$. Контур интегрирования $\gamma$ представлен на рис. 3 .

Проводя вычисления, аналогичные вычислениям для модельного интеграла, получим после УФ-регуляризации "нефизических" античастиц

$$
n_{0}=\frac{g_{s}}{\pi} \int_{0}^{\infty} \frac{z d z}{e^{\beta(z-\mu)}+1} .
$$

Выражение (21), в отличие от модельного интеграла, содержит кроме разреза дополнительный полюс П, связанный с наличием в знаменателе вклада $p_{0}+\mu-\varkappa \operatorname{ctg} \vartheta$ (напомним, что “физический” лист двузначной функции $\varkappa$ определяется условием $\varkappa>0$ при положительных $x)$. Этот полюс в точке $z=p_{y} \cos \vartheta$ на плоскости переменной $z=p_{0}+\mu$ мы изобразили на рис. 4 ; в случае $\vartheta=0$ он стремится к точке ветвления $p_{y}$.

После замены $z=p_{0}+\mu$, учета четности подынтегрального выражения по $p_{y}$ и деформации контура к вещественной оси граничный вклад профиля плотности принимает вид

$$
n_{\mathrm{b}}=\frac{g_{s}}{\pi^{2}} \oint_{\gamma_{+}^{\prime}+\gamma_{-}^{\prime}+\gamma_{\Pi}} \frac{d z}{e^{\beta(z-\mu)}+1} \int_{0}^{\infty} d p_{y} \frac{p_{y}^{2}}{\sqrt{z^{2}-p_{y}^{2}}} \frac{e^{-2 i x \sqrt{z^{2}-p_{y}^{2}}}}{z-i \sqrt{z^{2}-p_{y}^{2}} \operatorname{ctg} \vartheta},
$$

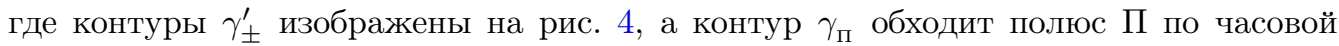
стрелке.

Таким образом, в граничные эффекты дают вклад частицы и физические дырки (вклад от контура $\gamma_{+}^{\prime}$ ), "нефизические" античастицы (вклад от контура $\gamma_{-}^{\prime}$ ) и связанные состояния, описываемые вкладом от контура $\gamma_{п}$. Ответы для вкладов, связанных с $\gamma_{+}^{\prime}$ и $\gamma_{-}^{\prime}$, не сложно преобразовать к виду

$$
n_{\mathrm{b} \pm}(x)=-\left.\frac{2 g_{s}}{\pi^{2}} \int \frac{z d z}{e^{ \pm \beta(z \mp \mu)}+1}\left(B(s, \vartheta) \mp \operatorname{ctg} \vartheta \frac{\partial B(s, \vartheta)}{\partial s}\right)\right|_{s=2 x z},
$$

где верхние знаки соответствуют контуру $\gamma_{+}^{\prime}$, нижние - контуру $\gamma_{-}^{\prime}$ и введена функция

$$
B(s, \vartheta)=\int_{0}^{1} \frac{d t \sqrt{1-t^{2}} \cos (s t)}{1+t^{2} \operatorname{ctg}^{2} \vartheta} .
$$

При $\vartheta=\pi / 2$ функция $B(s)$ выражается через функцию Бесселя:

$$
B(s, \pi / 2)=\int_{0}^{1} d t \sqrt{1-t^{2}} \cos (s t)=\frac{\pi}{2 s} J_{1}(s) .
$$

Вклад от интегрирования по контуру $\gamma_{\text {п }}$ сводится к

$$
n_{\Pi}(x)=\frac{2 g_{s}}{\pi} \int_{0}^{\infty} d p_{y} \underset{\Pi}{\operatorname{res}} \frac{p_{y}^{2} e^{-2 \varkappa x}}{\left(e^{\beta(z-\mu)}+1\right) \varkappa(z-\varkappa \operatorname{ctg} \vartheta)} .
$$

В рассматриваемом полюсе $\varkappa$ обращается в $p_{y} \sin \vartheta$, а функция $(z-\varkappa \operatorname{ctg} \vartheta)^{-1}$ имеет вычет $\sin ^{2} \vartheta$, поэтому

$$
n_{\Pi}(x)=\frac{2 g_{s} \sin \theta}{\pi} \int_{0}^{\infty} p_{y} d p_{y} \frac{e^{-2 p_{y} x \sin \theta}}{e^{-\beta\left(p_{y} \cos \theta+\mu\right)}+1} .
$$


При $0 \leqslant \vartheta<\pi / 2$ это выражение сводится заменой $p_{y} \cos \vartheta=z$ к интегралу

$$
n_{\Pi}(x)=\frac{2 g_{s} \sin \vartheta}{\pi \cos ^{2} \vartheta} \int_{0}^{\infty} z d z \frac{e^{-2 z x \operatorname{tg} \vartheta}}{e^{-\beta(z+\mu)}+1},
$$

который должен быть отброшен в рамках обсуждавшегося выше УФ-обрезания.

Заметим, что в случае граничного условия в приближении "бесконечной массы" $(\vartheta=0)$ не только $n_{\text {п }}$ не дает вклада в ответ, но и вклады $n_{\mathrm{b} \pm}$ обращаются в ноль. В этом, впрочем, несложно убедиться непосредственно, поскольку след слагаемого в пропагаторе $(9)$, содержащего $\Delta_{M}$, тождественно обращается в ноль. Однако при $\vartheta=\pi / 2$ выражение (27) дает нетривиальный вклад

$$
\left.n_{\Pi}(x)\right|_{\vartheta=\pi / 2}=\frac{g_{s}}{2 \pi x^{2}\left(e^{-\mu \beta}+1\right)} .
$$

В общем случае электронная плотность задается суммой $n_{0}+n_{\mathrm{b}+}(x)$ выражений (22) и (24); слагаемое (28) следует добавить в случае зигзагоподобного края графена $(\vartheta=\pi / 2)$.

Полученные результаты демонстрируют наличие дальнодействия при $\vartheta=\pi / 2$. Для остальных значений $\vartheta$ толщина $\delta$ характерного слоя, в котором существенны граничные эффекты, является функцией трех (безразмерных) параметров $\beta, \mu$ и $\vartheta$; численное значение $\delta$ при заданных $\beta, \mu$ и $\vartheta$ легко вычисляется при помощи явных формул $(22),(24)$. Так как профиль плотности вычислялся в обезразмеренных переменных, при неспецифических значениях этих параметров, когда $\delta(\beta, \mu, \vartheta) \sim 1$, характерная толщина этого слоя определяется размерным параметром $x_{\mathrm{F}}$.

\section{8. ЗАКЛЮЧЕНИЕ}

В то время как построенный нами пропагатор имеет регулярное поведение при любых значениях параметра $\vartheta$, сконструированная из него плотность числа частиц, как и ожидалось, отражает неаналитичность перехода к случаю $\vartheta=\pi / 2$.

При описании электронной плазмы в графене при конечной температуре и фиксированном химическом потенциале в рамках метода температурных функций Грина модель приобретает специфические черты по сравнению с родственной задачей о статистическом поведении ультрарелятивистского ферми-газа. Мы показали, что границы применимости модели связаны с введением УФ-обрезания модели ультрарелятивистского ферми-газа.

Полученный пропагатор температурных функций Грина можно использовать для построения регулярной теории возмущений при описании электронных свойств графена, связанных с взаимодействием типа "плотность-плотность", а также для описания фазовых переходов в данной системе.

\section{Список литературы}

[1] P. R. Wallace, "The band theory of graphite", Phys. Rev., 71:9 (1947), 622-634.

[2] C. Oshima, A. Nagashima, "Ultra-thin epitaxial films of graphite and hexagonal boron nitride on solid surfaces", J. Phys., 9:1 (1997), 1-20.

[3] K. S. Novoselov, A. K. Geim, S. V. Morozov, D. Jiang, Y. Zhang, S. V. Dubonos, I. V. Grigorieva, A. A. Firsov, "Electric field effect in atomically thin carbon films", Science, 306:5696 (2004), 666-669. 
[4] M.I. Katsnelson, Graphene. Carbon in Two Dimensions, Cambridge Univ. Press, Cambridge, 2012.

[5] C. G. Beneventano, I. Fialkovsky, E. M. Santangelo, D. V. Vassilevich, "Charge density and conductivity of disordered Berry-Mondragon graphene nanoribbons", Eur. Phys. J. B, 87:3 (2014), 50, 9 pp.

[6] C. G. Beneventano, E. M. Santangelo, "Boundary conditions in the Dirac approach to graphene devices", Internat. J. Modern Phys. Conf. Ser., 14 (2012), 240-249.

[7] A. R. Akhmerov, C. W. J. Beenakker, "Boundary conditions for Dirac fermions on a terminated honeycomb lattice", Phys. Rev. B, 77:8 (2008), 085423, 10 pp.

[8] А. А. Абрикосов, Л. П. Горьков, И. Е. Дзялошинский, Методы квантовой теории поля в статистической физике, Добросвет, М., 2006.

[9] А.Н. Васильев, Функционалъные методы в квантовой теории поля и статистике, ЛГУ, Л., 1976.

[10] A. Nieto, "Evaluating sums over the Matsubara frequencies", Comput. Phys. Commun., 92:1 (1995), 54-64. 